\title{
The Possibility of a Simultaneous Transmission of Ultrasound and Laser Radiation via Flexible Optical Silica Glass Fibre
}

\author{
S. Muc*, T. Gudra And E. Beres-PaWlik \\ Institute of Telecommunications, Teleinformatics and Acoustics, Wrocław University of Technology \\ Wybrzeże Wyspiańskiego 27, 50-370 Wrocław, Poland
}

\begin{abstract}
Glass optical fibres are a promising medium for simultaneous laser-ultrasonic applications. The proposed application system is based on simultaneous transmission of laser radiation and ultrasounds in a flexible silica glass fibre. The optical fibre's core was made of $\mathrm{SiO}_{2}(97 \%)$ and $\mathrm{GeO}_{2}(3 \%)$ and the cladding was $100 \% \mathrm{SiO}_{2}$. The material the optical fibre is made of enables simultaneous transmission of laser radiation and ultrasonic wave. Experiments were performed using a Mach-Zehnder optical waveguide interferometer with single-mode optical fibre coupler. This paper presents measurement results for delivering ultrasonic waves to the optical fibre using longitudinal vibrations generated by a sandwich ultrasonic transducer with a velocity transformer. The study presents the relations concerning simultaneous operation of both types of waves and the possibilities of transmission of low frequency, high power ultrasonic wave in optical fibres using a sandwich type transducer.
\end{abstract}

PACS numbers: 43.35.Yb, 42.81.Wg

\section{Introduction}

The Mach-Zehnder optical fibre interferometer is a well-known system widely covered in specialist literature [1-5]. A lot is also known about the relations involved in acoustic wave propagation in an optical fibre and through similarity to widely known optical formulas, the acoustic condition related to guiding acoustic waves in optical fibres [6-11]. According to the authors' information, however, there are no works related to simultaneous transmission of laser radiation and ultrasonic waves in optical fibres with the use of a power sandwich ultrasonic transducer. The following study results are to help to provide information on whether ultrasounds can affect the parameters of light wave propagating in an optical fibre and present the interaction of both types of waves.

\section{Experimental results}

The experimental study of simultaneous transmission of laser radiation and ultrasonic wave was performed using a Mach-Zehnder optical waveguide interferometer. A low frequency, high power ultrasonic wave, generated by a power sandwich transducer, was supplied to a signal arm. Ultrasonic vibrations introduce changing stress in the waveguide. Light modulation effect in the shape of a signal with frequency modulated by ultrasonic wave

\footnotetext{
* corresponding author; e-mail: Sylwia.Muc@pwr.wroc.pl
}

(light wave phase modulated) was observed on the oscilloscope screen. As a result of light modulation it is possible to observe interference fringes, which are detected using a PIN diode and are a characteristic of changing phase in a Mach-Zehnder interferometer system (Fig. 1). In a single-mode waveguide, acoustic wave propagates in the core and in the cladding [12].

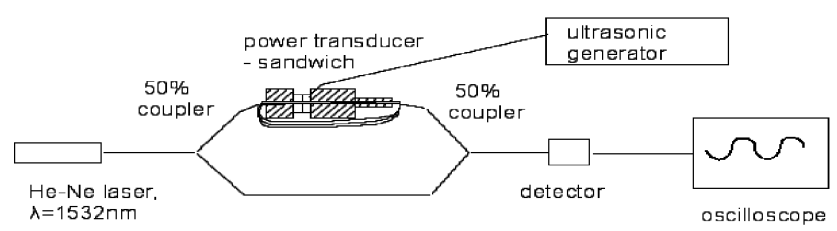

Fig. 1. Mach-Zehnder interferometer with a power sandwich ultrasonic transducer.

In the course of the study it was used an AG Series power amplifier by T\&C Power Conversion controlled by means of a HP 33120A generator, which enables the display of forward (FP), reflected (RP) and load (LP) power. The frequency band in which the interference fringes were achieved for the sandwich type transducer is presented in Fig. 2. The transducer operates at the resonance frequency of $52.5 \mathrm{kHz}$. The observed interference fringes appear in the resonance frequencies band. The measurements were also performed for 3 turns of the optical fibre around the transducer (the method presented in Fig. 1) in order to monitor the changes in bandwidth in relation to the number of turns. Analysing information 


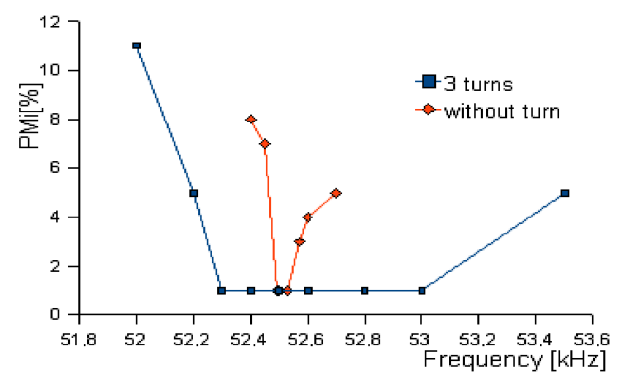

Fig. 2. Delivered power mode input (PMi) versus frequency.

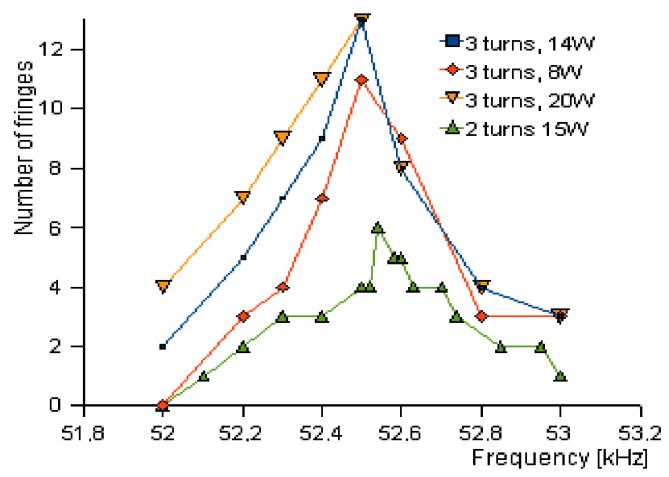

Fig. 3. Dependence of the number of fringes on frequency.

in Fig. 2 allows conclusion that the more turns the larger the bandwidth in which the fringes can be observed.

Figure 3 shows the relation of the number of fringes to the number of turns around the sandwich type transducer for various values of power delivered to the transducer. The number of fringes is the largest for the res-

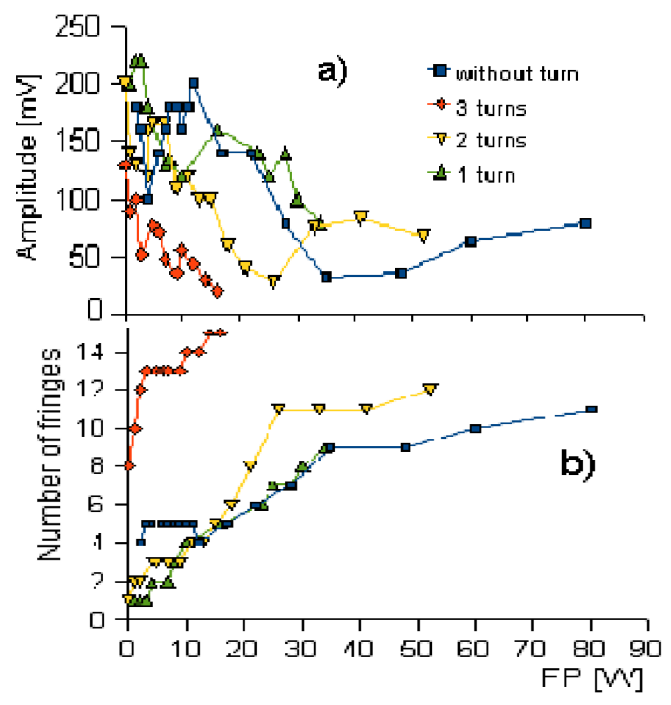

Fig. 4. Relation of (a) amplitude, (b) the number of fringes, to the power supplied to the transducer. onance frequency. Since the number of fringes does not depend on frequency, it means that for resonance frequency the phase change is the most significant. Additionally, analysis of the relations presented in Fig. 3 leads to the conclusion that increase in power causes increase in the number of fringes, which means that bigger phase change occurs. The more fibre turns, the more fringes are observed (which in turn indicates bigger phase change).

The investigations were conducted for power delivered to the transducer in a range of $0 \div 80 \mathrm{~W}$ (Fig. 4). Figure 4 shows the relation of output signal amplitude from the detector to the power supplied to the sandwich type transducer $\left(f_{\text {res }}=52.5 \mathrm{kHz}\right)$. When power increases, the amplitude decreases (Fig. 4a) and the number of fringes rises (Fig. 4b). In the ranges in which the amplitude increases together with power (Fig. 4a), the number of fringes remains constant. In order to fully explain this phenomenon it will be necessary to perform additional

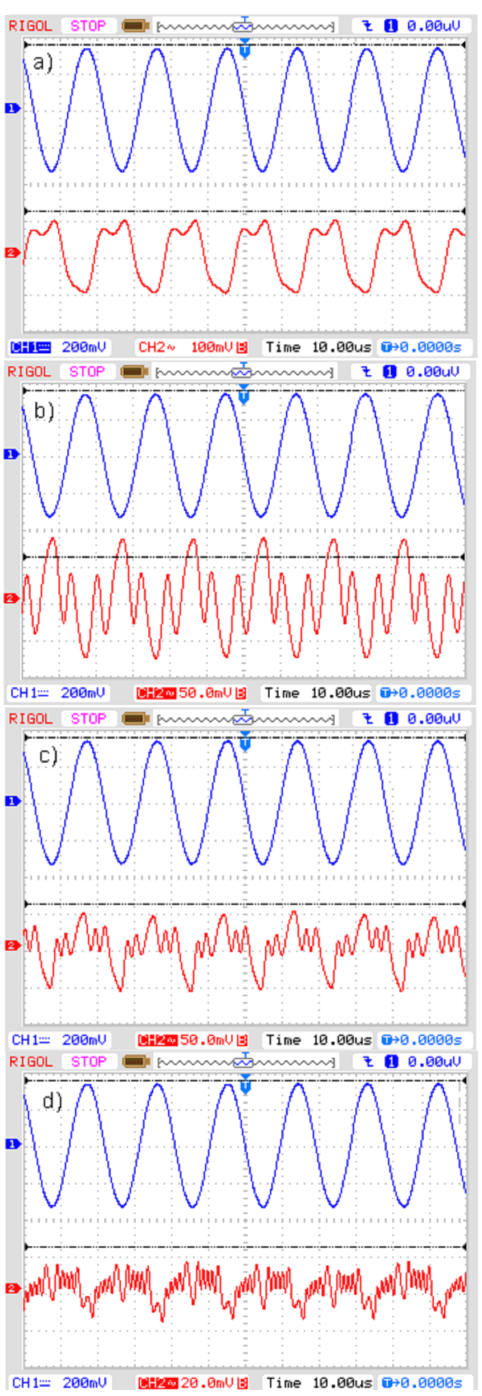

Fig. 5. Dependence of the number of stripes on power: (a) $0.4 \mathrm{~W}$, (b) $4 \mathrm{~W}$, (c) $12 \mathrm{~W}$, (d) $22 \mathrm{~W}$. 
experiments, but it can be initially assumed that for resonance frequencies, phase change gets bigger at the cost of decreasing amplitude.

Figure 5 presents waveforms obtained on the oscilloscope screen, for $f=52.5 \mathrm{kHz}$, which shows the number of fringes increasing together with power.

It is a great benefit in the field of monitoring a power delivery system. It is possible to determine how much power is delivered to the transducer by measuring reflected power. Reflected power for the resonance frequency of $52.5 \mathrm{kHz}$ is insignificant (Fig. 6a). The transducer also has a second resonance $(f=50.6 \mathrm{kHz})$, for which the reflected power is higher (Fig. 6b) and the transducer effectiveness lower. Tuning slightly away from the resonance frequency (Fig. 6c) is enough to observe a significant increase in the reflected power (high power loss). This is why it is important to guarantee frequency stabilization.

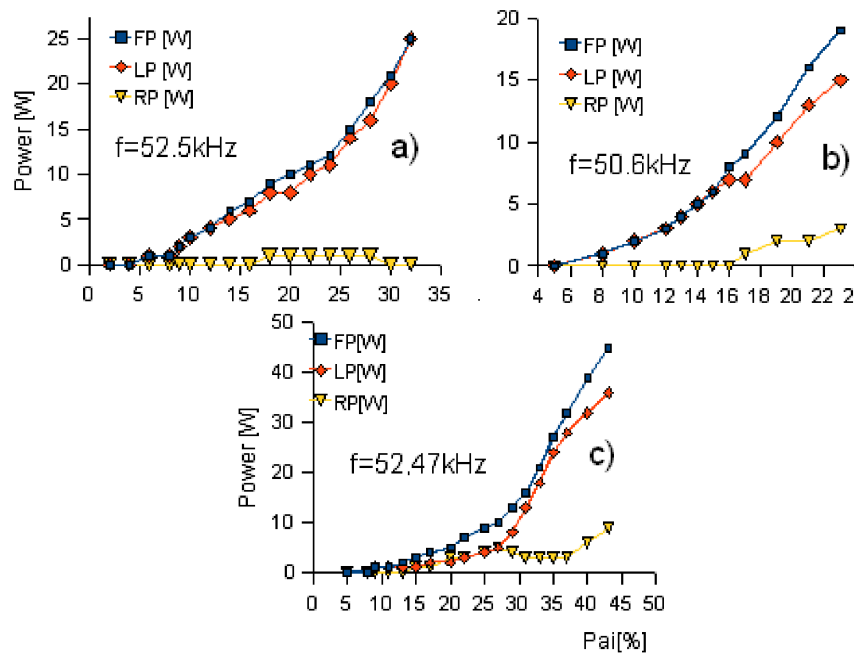

Fig. 6. Relation of reflected power to frequency, (a) $f_{\text {res } 1}=52.5 \mathrm{kHz}$, (b) $f r_{\text {res } 2}=50.6 \mathrm{kHz}$, (c) frequency beyond resonance.

The number of fringes can be used to calculate the displacement amplitude of an optical fibre $(D)$. One fringe corresponds to half of the length of light wave [13-17]. Displacement amplitudes of the optical fibre were specified on the basis of the graphs presented in Fig. 7. Increase in power and the number of coils results in increase in the amplitude of this displacement. In order to precisely determine the displacement amplitude (in $\mu \mathrm{m}$ ) it will be necessary to use a laser vibrometer or a capacitive displacement sensor. For example, in an ultrasonic surgical knife constructed on the basis of sandwich transducer displacement amplitudes of the knife tip remain in the range of $10 \div 130 \mu \mathrm{m}$. It is possible to increase displacement amplitude by increasing the number of coils and/or power supplied to the transducer.

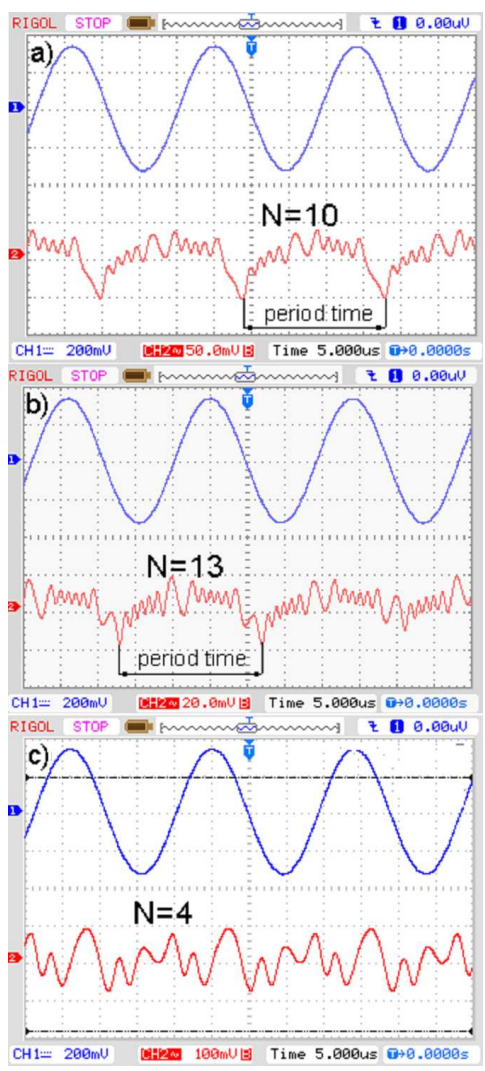

Fig. 7. The relation of the number of fringes $(N)$ to the number of turns, $f=52.5 \mathrm{kHz}$, (a) 3 turns $P=1 \mathrm{~W}$, $D=7.66 \mu \mathrm{m}$, (b) 3 turns, $P=7 \mathrm{~W}, D=9.96 \mu \mathrm{m}$, (c) without turn $P=8 \mathrm{~W}, D=3.06 \mu \mathrm{m}$.

\section{Conclusion}

The work presents the possibility of transmitting ultrasonic wave, which is generated by a sandwich ultrasonic transducer, in an optical fibre. The obtained results will serve as material for further research related to the analysis of the possibilities of simultaneous transmission of laser radiation and ultrasonic waves in optical fibres $[18,19]$. The results, together with the performed initial theoretical analysis of the possibilities of compensating the disadvantages of laser and ultrasonic technologies in surgery [20, 21], lead to the conclusion that it is possible to combine the advantages of a laser and ultrasonic surgical knife in one device.

\section{References}

[1] K.T.V. Grattan, B.T. Meggitt, Optical Fiber Sensor Technology, Chapman \& Hall, London 1995.

[2] D.A. Jackson, J.D.C. Jones, Optica Acta 33, 1469 (1986).

[3] E. Udd, Fiber Optic Sensors: An Introduction for Engineers and Scientists, Wiley, New York 2006.

[4] A. Dandridge, Proc. SPIE 1985, 34 (1998).

[5] D.A. Jackson, J. Phys. E. Sci. Instrum. 18, 981 (1985). 
[6] D. Nghambia Mbamou, J. Helfmann, G. Müller, G. Brunk, T. Stein, K. Designer, Meas. Sci. Technol. 12, 1631 (2001).

[7] C.K. Jen, in: IEEE Ultrasonics Symp. 1985, p. 1128.

[8] C.K. Jen, in: IEEE Ultrasonics Symp. 1987, p. 443.

[9] C.K. Jen, A. Safaai-Jazi, G.W. Farnell, in: IEEE Ultrasonics Symp., 1986, p. 1113.

[10] A. Safaai-Jazi, C.K. Jen, G.W. Farnel, IEEE Trans. Ultrason. Ferroelectr. Freq. Control. 33, 526 (1986).

[11] A. Safaai-Jazi, R.O. Claus, IEEE Trans. Ultrason. Ferroelectr. Freq. Control. 35, 262 (1988).

[12] S. Muc, Arch. Acoust. 33, 619 (2008).

[13] T.K. Gangopadhayay, P.J. Henderson, Appl. Opt. 38, 2471 (1999).

[14] M. Gawlikowski, B. Przywara-Chowaniec, T. Pustelny, P. Struk, Mol. Quant. Acoust. 28, 329 (2007).
[15] T.K. Gangopadhayay, P.J. Henderson, Meas. Sci. Technol. 16, 1075 (2005).

[16] T.K. Gangopadhayay, Meas. Sci. Technol. 15, 911 (2004).

[17] N. Sathitanon, S. Pullteap, Int. J. Computer Sci. Eng. 2, 63 (2007).

[18] P. Gibinski, E. Maciak, Z. Opilski, T. Pustelny, A. Sieron, M. Urbanczyk, Mol. Quant. Acoust. 28 , 217 (2007).

[19] M. Gawlikowski, T. Pustelny, R. Kustosz, Eur. Phys. J.-Special Topics 154, 63 (2008).

[20] T. Gudra, S. Muc, Arch. Acoust. 32, Suppl., 117 (2007).

[21] T. Gudra, S. Muc, Eur. Phys. J.-Special Topics 154, 85 (2008). 